\title{
Therapie muskuloskelettaler Erkrankungen - Stand und Perspektiven
}

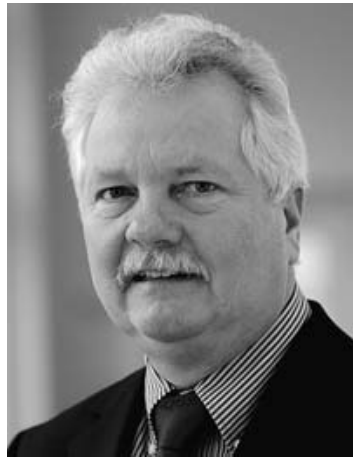

G.-R. Burmester

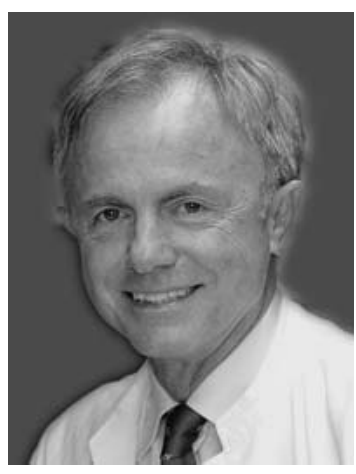

S. Endres

Bibliografie

Dol http://dx.doi.org/ 10.1055/s-0035-1558110

Drug Res 2015; 65, Suppl. 1: S1

(c) Georg Thieme Verlag KG

Stuttgart · New York .

ISSN 0004-4172

Korrespondenzadresse

Prof. Dr. Gerd-Rüdiger

Burmester

Charité Universitätsmedizin

Berlin

Medizinische Klinik mit

Schwerpunkt Rheumatologie

und Klinische Immunologie

Charitéplatz 1

10117 Berlin

gerd.burmester@charite.de

\section{Prof. Dr. Stefan Endres}

Ludwig-Maximilians-Universität

München

Abteilung für Klinische

Pharmakologie

Ziemssenstraße 1

80336 München

endres@Imu.de
In Deutschland sind rund 30 Mio. Menschen von muskuloskelettalen Erkrankungen betroffen. Dazu gehören insbesondere entzündlich-rheumatische Erkrankungen, Arthrose und Osteoporose sowie Rückenschmerzen. In einer Gesellschaft des immer längeren Lebens und aufgrund des bewegungsarmen Lebensstils ist mit einer weiteren Zunahme dieser Erkrankungen zu rechnen: Neben direkten Folgen wie chronischen Schmerzen, Funktionseinschränkungen und damit Verlust an Lebensqualität haben diese Krankheiten auch erhebliche volkswirtschaftliche Konsequenzen, da sie zu den häufigsten Anlässen für Arbeitsunfähigkeit und Frühverrentung zählen. Darüber hinaus sind insbesondere bei den entzündlich-rheumatischen Erkrankungen, bei denen autoimmunologische und autoinflammatorische Aspekte eine entscheidende Rolle spielen, wichtige diagnostische und therapeutische Fortschritte gemacht worden.

Grund genug, den Stand und die Perspektiven der Therapie muskuloskelettaler Erkrankungen umfassend zu beleuchten! In einigen Bereichen hat es zudem in den letzten Jahren Fortschritte in der molekularen Aufklärung der zugrunde liegenden Krankheitsmechanismen gegeben. So werden u.a.
Erfolge und Herausforderungen bei Rheumatoider Arthritis und Spondyloarthritiden sowie neue Perspektiven für Arthrose- und Osteoporose-Therapie vorgestellt. Ein weiterer Schwerpunkt liegt auf neuen pathogenetischen Konzepten, auf deren Basis sich weitere Therapiemöglichkeiten u. a. bei der Sarkopenie und bei Myositiden eröffnen könnten.

Schließlich wird es auch darum gehen, wie die Rheumatologie neue Erkenntnisse und Behandlungsmöglichkeiten aus der Onkologie für ihr Gebiet nutzen kann, und welche Fortschritte es bei Muskeldystrophien gibt.

Dem Zweck der Paul-Martini-Stiftung entsprechend sollen bei diesem Symposium etablierte Therapien sowie Forschungs- und Entwicklungsarbeiten im Bereich der akademischen Forschung und der Industrie präsentiert und von den Teilnehmern aus Wissenschaft und Industrie, aber auch aus anderen Bereichen des Gesundheitswesens diskutiert werden.

\section{Gerd-Rüdiger Burmester \\ Charité Universitätsmedizin Berlin \\ Stefan Endres \\ Ludwig-Maximilians-Universität München}

